\title{
Mining data from intensive care patients
}

\author{
Jan Ramon ${ }^{\mathrm{a}, *, 1}$, Daan Fierens ${ }^{\mathrm{a}, 2}$, Fabián Güiza ${ }^{\mathrm{a}, 3}$, Geert Meyfroidt ${ }^{\mathrm{b}}$, \\ Hendrik Blockeel ${ }^{a}$, Maurice Bruynooghe ${ }^{a}$, Greet Van Den Berghe ${ }^{b}$ \\ ${ }^{a}$ Department of Computer Science, Katholieke Universiteit Leuven, Belgium \\ ${ }^{\mathrm{b}}$ Department of Intensive Care Medicine, University Hospital, Leuven, Belgium
}

Received 24 October 2006; accepted 22 December 2006

\begin{abstract}
In this paper we describe the application of data mining methods for predicting the evolution of patients in an intensive care unit. We discuss the importance of such methods for health care and other application domains of engineering. We argue that this problem is an important but challenging one for the current state of the art data mining methods and explain what improvements on current methods would be useful. We present a promising study on a preliminary data set that demonstrates some of the possibilities in this area.
\end{abstract}

(C) 2007 Elsevier Ltd. All rights reserved.

Keywords: Intensive care; Dynamic systems

\section{Introduction}

Critically ill patients need intensive care for their survival. In an intensive care unit (ICU), the vital functions of patients are being monitored continuously. In order to save a patient's life, these functions can be supported by medications or by a mechanical device when threatened, until the patient is able to perform these functions again autonomously. For example a patient with respiratory failure can be mechanically ventilated or a patient with kidney failure

\footnotetext{
* Corresponding author. Tel.: +32 486968518; fax: +3216327996.

E-mail addresses: Jan.Ramon@cs.kuleuven.be (J. Ramon), Daan. Fierens@cs.kuleuven.be (D. Fierens), Fabian.Guiza@cs.kuleuven.be (F. Güiza), Geert.Meyfroid@uz.kuleuven.be (G. Meyfroidt), Hendrik.Blockeel@cs.kuleuven.be (H. Blockeel), Maurice.Bruynooghe@cs.kuleuven.be (M. Bruynooghe), Greta.Vandenberghe@med.kuleuven.be (G. Van Den Berghe).

1 Jan Ramon and Hendrik Blockeel are post-doctoral fellows of the Fund for Scientific Research (FWO) of Flanders.

${ }^{2}$ Daan Fierens is supported by the Institute for the Promotion of Innovation through Science and Technology in Flanders (IWT Vlaanderen).

${ }^{3}$ Fabián Güiza and Geert Meyfroidt are supported by the Interdisciplinary Research Fund of the Katholieke Universiteit Leuven (IDO 3M030483)
}

can be supported with hemodialysis or hemofiltration. Although several major breakthroughs in patient care, monitoring systems and therapeutic modalities have led to an improved survival rate for ICU patients, many challenges remain. While $70 \%$ of the ICU patients need vital support and monitoring only for a few days and have a high chance of survival, $30 \%$ of the patients stay in the ICU for a longer period, sometimes even months. For these prolonged critically ill patients, the likelihood of dying raises as their length of stay increases (e.g. after 3 weeks the likelihood is $30 \%$ ). These patients show a uniform picture of an immune system out of balance. Their immune system is depressed making them more subject to severe infections, or it is overactive and leads to a hyper-inflammatory state which threatens vital organ systems such as the lungs, the heart, the kidneys, the liver or the brain. Sometimes, in spite of maximal effort, this multiple organ failure cannot be resolved (even after weeks or months), leading to death of the patient. Current risk prediction models in intensive care $[29,13,14,17,19]$ were designed to predict the risk of mortality or complications for patient populations, but are not accurate enough for prediction for individual patients. Currently no tools exist to reliably predict an individual patient's chance of developing a complication such as organ failure. 
There is already a lot of literature on data mining for ICU. On the one hand there are articles aimed at medical doctors and other experts in intensive care, explaining the range of data mining methods that are currently available and/or illustrating on specific problems how they can be useful $[21,16,15,31]$. On the other hand, there are articles aiming also (or mainly) at an audience from machine learning or statistics $[25,24,10]$, introducing specific problems in intensive care and how they can be solved with data mining techniques. Thus, there are articles that focus on concrete problems and solutions, and articles that introduce a range of solutions, but to our knowledge there are none that describe a range of challenges that need to be addressed. The latter would be interesting because, apart from saying what can be done for ICU with data mining, it is obviously useful to say what cannot be done in a satisfactory way right now, so that the machine learning community can focus on developing new solutions.

This article attempts to fill this gap. While it is not our intention to provide an exhaustive overview of all the challenges that ICU data mining poses, our own experiences on a specific dataset have yielded a list of challenges that seem worth looking at. In a first part of the paper we will discuss ICU data mining on a general level, listing the challenges that we see as well as possible directions for addressing them. In a second part of the paper we describe in some more detail our own experiments, which illustrate the points made before and show the potential of, as well as the problems faced by, current data mining approaches. We include research prototypes of cutting-edge data mining technology and well as standard data mining methods.

From an engineering point of view, the reported research is not only important for the ICU where it helps in the challenging task of optimising the available technical means to maximise the health of patients. It is more generally applicable to the study of living organisms (such as growing plants and animals on an industrial scale). Moreover, this research can help in assessing the added value of supporting and monitoring systems and possibly in a further development of these. Finally, similar techniques as used for predicting cohorts of patients can be used in other planning and scheduling problems in the field of engineering.

The remainder of this paper is structured as follows. In Section 2 we will describe the application, the data present in a typical intensive care setting, and the challenges of an intensive care unit. In Section 3 we will discuss approaches for building models in intensive care. Next, in Section 4 we will discuss the challenges we see for the data mining community. We will describe our preliminary study on a smaller data set in Section 5. We will conclude in Section 6 and outline our further work.

\section{Intensive care}

\subsection{The application}

Critically ill patients are patients in need of vital monitoring and/or support. People can need intensive care after major surgery, e.g. cardiac surgery. Also, solid organ transplant patients will be critically ill in the first days or weeks after their transplantation. People can be admitted urgently or unplanned to an intensive care unit, e.g. after a major trauma, severe burns, an overwhelming infection, an acute organ failure, an exacerbation of a pre-existing chronic disease. Of course, there is a wide range of severity of critical illness. Some patients need only monitoring and minor adaptations to their medication therapy, others need full support and monitoring. Because a lot of these patients are no longer able to take care of their basic needs, support of e.g. nutrition by artificially feeding these patients through intravenous infusion or infusion in the gastrointestinal system is often necessary.

\subsection{Available data}

A wide range of data is available, but usually not in an integrated form. The central information of an ICU is of course the data on patients, of which we can distinguish the following:

- demographic data: For every patient, demographic information (birth date, sex, address, etc.) is collected. Most of this data can be assumed to remain unchanged during the stay in the ICU.

- Historical DATA: Every patient may also have a medical history of previous illnesses and treatments, which may be relevant for decisions about treatment in the future. Unfortunately, this data is not always available, e.g. in the case of unplanned ICU admissions or due to the confidentiality of certain medical information.

- ICU STAY DATA: This data forms a chronological record of the stay in the ICU. It includes regularly registered information about the state of the patient, and information about every treatment of the patient in the broadest sense. The first category can include

- PARAMETER MEASUREMENTS: measurements of clinical parameters (e.g. temperature, blood pressure, heart rate, etc.).

- LABORATORY DATA: Laboratory results, obtained by examination of samples from the patient in a lab.

- BACTERIOLOGICAL DATA: Information about infections of patients.

- SUBJECTIVE OBSERVATIONS: Records of subjective observations of doctors and nurses about the patient (e.g. based on skin colour or other difficultly quantifiable observations). It has been observed that despite the huge effort in creating objectively measurable parameters and in standardisation, subjective observations remain very important (see also Section 5.7.1).

The latter category concerning treatment information can include

- MEDications: data about medications administered to the patient. This data can be divided into two categories depending on the method of administration: bolus medication (administered at a particular point 
in time) and continuous medication (administered over a period in time in a continuous way).

- TREATMENTS: information about treatments the patient receives. Similarly, this can be divided into continuous treatments (e.g. mechanical ventilation, hemofiltration, etc.) and treatments at a particular time point or in a short time period (e.g. hemodialysis, surgery, etc.).

- FEEDING: Records of how much and which kind of food the patient receives.

- TREATMENT POLICY DECISIONS: Depending on the state of the patient and the evolution observed, doctors may decide at a given point in time to follow a particular treatment in the future for this patient. This may or may not have an immediate effect on the treatment itself.

Apart from patient data, several other data sources may be available and valuable for data analysis and decision making. In this paper we will call this information background data. This includes

- medication information: Data on the known effects of medications and the typical situations in which and the reasons why they are applied.

- EXPERT KNOWLEDGE: Partial knowledge from experts, e.g. describing the interpretation of (combinations of) symptoms, models of parts of the functioning of the human body, known interactions between medications or risks of treatments, etc.

Data collection challenges are twofold. First, the task of collecting the data for each of the categories described above is quite work-intensive. Even for those categories where automated collection of data is possible, it is necessary to check the correctness of it. Second, the integration of the different data sources is often difficult, due to the heterogeneous platforms and formats used to create this data, standardisation problems, confidentiality, etc.

Recently, many intensive care units are installing a patient data management system (PDMS) that allows both online data collection and a database system where data from several sources is integrated. These approaches can greatly help to address both of the above problems.

\subsection{ICU challenges}

Crucial in intensive care is to provide the correct treatment and to detect clinical problems early enough so preventive or curative measures can be taken in time. If one can detect early that a patient's state is worsening, the chance of a successful treatment is higher. In practice, an intensivist analyses all the patient related data (obtained by clinical examination, patient monitors, laboratory analysis, technical examinations (such as radiology), the response of a patient to his treatment, etc.), in order to detect a change in the condition of his patients. A decreasing trend in the functioning of a particular organ could indicate that there is a risk that this organ will fail in the near future. However, the data may contain much more information than one can currently extract from it. Human intellectual capacity is simply not capable of handling more than 5-7 different parameters at the same time [22]. In the early eighties already, it was estimated that an average ICU patient is described by approximately 250 different parameters.

Data mining could assist clinicians, analysing the data from ICU patients and detecting problems earlier than an experienced intensivist would. The challenge then becomes twofold: first learn which patterns indicate which problems (i.e. learn to recognize the problem when it happens and in this way find patterns describing the diagnosis) and second learn to predict these patterns in the huge amount of data available as early as possible.

Once problems are detected, they should be solved by taking the appropriate actions (e.g. administer a treatment). Whether or not a certain action should be taken given the predicted risks depends on the cost and potential benefit of the action. Developing a strategy that optimises the cost/benefit trade-off is a non-trivial task. In this paper we consider only the problem of predicting risks. Developing a strategy that exploits these predictions and evaluating its benefit is considered future work.

\section{Approaches to gaining domain knowledge in ICU}

In the previous section we have explained that a large amount of data is available in the intensive care domain. We have also stated that the current knowledge and decision making expertise is incomplete and can be improved. Several approaches can be tried to use the data in helping to improve this expertise. In this section we give an overview of directions considered in the past.

Methods can be either symbolic or subsymbolic. We say a method is symbolic if the models it produces can be interpreted and can therefore improve the insight in the domain. A typical example of such a method is the use of decision trees which we will apply in Section 5. Often however, methods that provide the most accurate results are subsymbolic. For example a very complicated formula or a support vector machine may accurately predict a particular target attribute, without providing much insight. Both kinds of methods have their merits in the intensive care domain.

One can classify the approaches depending on the way their models are generated. A first strategy, the construction of hand-crafted models, is quite popular in intensive care research [5]. These models are usually intended to describe a small part of the functioning of the human body. For example [36] reviews specific models for the inflammatory response. They get complex quite quickly, e.g. models using 20 differential equations to describe one phenomenon are not exceptional. For a more global or longer term picture, the detailed knowledge to build a mechanistic model is often lacking. A second strategy can be applied here: the analysis of data to generate statistical patterns. These 
models make abstraction of a lot of low-level details and focus on what they want to explain or predict. As experts have partial domain knowledge, especially methods combining knowledge gained from data and knowledge provided by experts are valuable.

Although to the best of our knowledge no systematic study has been conducted on a large intensive care dataset, in recent years several efforts have shown the interestingness of applying machine learning methods. One of them is the work $[23,24]$ which combines expert knowledge with statistical methods on a dataset of 148 patients which contains records of 118 attributes registered every minute (for a total of 679,817 records). The result is a system that recommends therapeutic interventions. A number of approaches specialise on learning about a particular body function (e.g. [10] analyses the respiratory pressure-volume curves of mechanically ventilated patients), a particular problem ([25] analyses hospital infections and [34,35] analyse threshold selection for models predicting length of stay in ICU) or considers a subpopulation of the intensive care patients (e.g. [1] builds a predictive model for the survival of patients that undergo damage control surgery).

Central to all these approaches is collection of data. In recent years, researchers started actively to collect data for analysis (see e.g. [30]). It is recommendable to integrate the collection of data with the daily routine of the intensive care unit, so that data can be used both by clinicians for their current patients and by researchers for analysis.

The growing number of papers in the critical care literature that surveys basic data mining methods ([21,16, 15,31]) shows the significant interest of researchers in this domain to apply data mining methods to improve their knowledge.

\section{Data mining challenges}

In this section we discuss a number of challenges data miners face in this domain. Even though many of these challenges are also present in other application areas, the combination of these general challenges and a number of specific characteristics not found in other applications (see e.g. Section 4.2) makes intensive care a very interesting application domain. For each of these challenges, we will explain how we think, based on our past experience, one could address them.

\subsection{Database size}

When using online monitoring systems, a huge amount of data is generated. Monitoring measurements are performed once per 1-3 min. About 20 attributes are measured, so we have about $20 \times 60 \times 24 \times 365=10,000,000$ measurements per bed per year. Our hospital has 56 beds in the surgical intensive care unit where online monitoring will be installed in the near future, which makes $500 \mathrm{M}$ measurements yearly, for about 2000 patients per year. Database administrators estimate that including all background information, indexes and administrative data, about $50 \mathrm{~GB}$ of disk space will be needed yearly. The total number of critical care beds in the hospital is currently 250 . The amount of data will increase by another order of magnitude when all 250 critical care beds will be monitored online. While this vast volume of data will challenge the processing capacities of data mining algorithms, it also creates opportunities to reach more reliable conclusions.

To address this problem, one plausible way is to structure the domain using domain experts' knowledge. This allows to represent the data at the right level of abstraction, which can reduce the data set size significantly. For example knowing the time scale on which certain processes happen can help to structure the time dimension. For example as an infection develops over hours or days, one should not search for patterns of parameters evolving within minutes or weeks. Another example is the drug database, where a taxonomy on the drugs (describing their use) can help to abstract away from the huge amount of different drugs.

\subsection{Individual characteristics of patients}

Usually, machine learning techniques try to learn a function from examples to class labels directly. However, patients have individual characteristics. It is often difficult to compare absolute values of attributes between patients. Let us consider a few examples.

- The values of many attributes are considered abnormal if they change significantly from the previous values of this particular patient, rather than if they deviate significantly from some universal value. For example the definition of acute kidney failure relates the current creatinine level to the level on entrance of the ICU instead of giving an absolute threshold for the creatinine level. Deviation from the universal absolute value, before ICU admission, is of course an important indicator of the patient's pre-existing kidney failure and can be an indicator of chronical kidney failure.

- Certain patients may react more, less, faster or slower to certain medications.

Therefore, apart from the 'global' learning over patients, another level of 'local' learning of characteristics of patients would be useful. While at the time of admission little is known about the patient, after a few days one will start to have sufficient statistics to start learning about the patient's characteristics.

To predict the evolution of patients, we intend to apply a two-level Bayesian approach to learn a model that depends on patient-specific parameters (e.g. instead of using a patient's current heartrate as an absolute value we want to use the deviation of his current heartrate w.r.t. his 'normal' heartrate). As a new patient arrives, a second learner will estimate these patient-specific parameters such as the 'normal' heartrate of the patient. Of course, at admission time, the patient is still unknown and these 
estimates will not be very accurate. But the longer one can observe the patient, the better one can estimate his patientspecific parameters and the better one can instantiate the global evolution-predicting model. It will be challenging to define a good set of patient-specific parameters. Nevertheless, we believe this approach is worth trying as it would both increase insight in what are good parameters characterizing patients, and put suitable structure and bias in the learning process.

\subsection{Noise}

The manually entered data used for our preliminary study is sometimes noisy or difficult to interpret and sometimes missing. Noise arises e.g. when different people write the name of a product in different ways, or use different names for the same medication, or use different medications with a similar working to treat the same problem. It turned out to be quite difficult to pre-process all medication records such that they would use unique names for the same active substance.

But also for the case of data produced by an automatic monitoring system, noise can be expected. For example it is known that moving the patient and especially changing his height w.r.t. certain machines may give a shift in the measured values. One strategy that is often followed to validate the online measurements is that a nurse checks the monitoring equipment at regular time intervals, and corrects if necessary. The result is a database with an unusual noise model: there are high resolution measurements, with low resolution validation points. If the measurement curves vary smoothly on crossing a validation point, one could conclude that the noise level in the time interval prior to the validation is probably negligible. On the other hand a discontinuity in the measurement curves after a validation point would be an indication of a corrective action on the nurse's part. This means that special care should be taken when handling the data in the time interval prior to this validation point.

As we are involved ourselves in the collection process of our data, we can make suggestions to minimize noise. For example the validation mechanism discussed above helps to isolate part of the unreliable data. Though a lot of noise is unavoidable, it helps also to be able to estimate the amount of noise from knowledge of the data collection process. Apart from these preventive measures, it is important to use noise-tolerant data mining approaches such as Bayesian methods and ensemble methods.

\subsection{Relational data}

As discussed in Section 2.2, the available data is multirelational. For example record with different primary key such as drugs and patients cannot be represented in a single table (see also [6]). Most existing (so-called propositional or attribute-value) data mining systems cannot optimally exploit relational knowledge. A first solution is to define smaller learning tasks that only use part of the data. However, this is not always sufficient. Therefore, multi-relational data mining methods (see e.g. [8]) are essential to deal with all the knowledge.

\subsection{Inductive knowledge bases}

Due to the large number of different but related prediction tasks, this application domain is a good example of a problem where an inductive knowledge base would be valuable. It is possible that several learning tasks can be solved easier using a model learned for other prediction tasks. For example when one has good predictors for predicting kidney failure and inflammation, one could use them as components in a model predicting survival. Also, when one has good models for the short-term evolution of a particular target attribute, it may become easier to build a long-term predictor. An inductive knowledge base is a system where the learned knowledge is added to the knowledge base and can serve as input for future learning.

We therefore aim at considering a range of prediction tasks together, where the different predictors can use each others opinion to make their own prediction. On the one hand, such a strategy allows to reuse learned knowledge, while on the other hand it avoids the difficulty to optimize a global model.

\section{A preliminary study}

In this section we will present a preliminary study on a small dataset with daily registered data. We use a heterogeneous dataset with patients admitted due to a variety of reasons. The aim of this study is two-fold. First, we want to illustrate with real data the discussions of the previous sections. Second, we consider several prediction tasks, some of which were not addressed with machine learning strategies before, but for which there is great interest in intensive care because of their importance in determining the outcome of a patient.

We illustrate the previous sections as follows. We investigate the difficulty of performing different prediction tasks on a real database. We want to see which tasks require complex methods to be solved and for which tasks simple algorithms perform well. Tasks on which good performance is obtained provide motivation to consider the more difficult scenario of predicting from high-resolution data from online-monitoring. Tasks on which poor performance is obtained highlight the challenging aspects of predicting in this domain and how current standard algorithms using a moderate-sized database of low-resolution data are not sufficient to address them.

\subsection{Setup}

The database used for our preliminary study had been previously created for a large randomised controlled trial studying the effects of intensive insulin therapy in critically 
ill patients [32]. The study showed that strictly controlling the blood sugar levels in a group of surgical intensive care unit patients reduced the mortality almost by half. This database contains data from 1548 mechanically ventilated adult patients admitted to our surgical ICU between February 2, 2000, and January 18, 2001. Written informed consent had been obtained from the closest family member and the study protocol was approved by the institutional review board. The patient population is described in detail in [32].

Upon inclusion of a patient in the original study, an admission record was created in the database. This record contains general information about the patient that is valid throughout the whole stay in ICU of the patient: age, sex, diagnosis, reason for admission, etc. (a complete description is given in Appendix A). Additionally, each day of a patient's stay in ICU a selection of vital parameters, laboratory results and information about therapy and medication administered to the patient were registered. This information was originally recorded in six tables of a relational database. However, certain parts of these tables could not be used as such for data mining: e.g. we had information about which drugs were administered to which patients but not enough information about the drugs themselves (purpose of drugs, equivalence of drugs with different names, etc.) to use this for data mining. Hence we decided to select all useful attributes from these six tables and combine them into a single new table with one record for each day of each patient's stay in the ICU (the selected attributes are given in Appendix A). This means that in total the data used for our preliminary study consists of two parts: 1548 admission records (one for each patient) and 11,834 daily records (one for each day of each patient's stay in the ICU).

\subsection{Application domain concepts}

Inflammation and organ failure are clinical conditions that severely affect the outcome of patients. In the medical literature, criteria have been developed to define states of elevated inflammation and kidney dysfunction $[20,2]$. These definitions are internationally accepted and have clinical relevance. Not all attributes needed for the definition were present in the database. Therefore, we used slightly different definitions, which are formally different from the standard ones but in practice analogous, and which could be computed from the available data.

- KIDNEY-DYSFUNCTION: A patient has kidney-dysfunction on a given day if at least one of the following three criteria is true for that patient on that day:

- creatinine in plasma is at least twice as large as its value on the first day in ICU (Creatinine is a degradation product from the muscle that is excreted by the kidneys. Because it is produced in a more or less constant way it can be used as a measure for kidney function),
- oliguria (urine output $<12(\mathrm{ml} / \mathrm{kg} / 24 \mathrm{~h}$ ); obviously, if the kidneys produce less urine it is a sign of dysfunction),

- new dialysis: either intermittent hemodialysis or continuous veno-venous hemofiltration is started.

- inflammation (in analogy to systemic inflammatory response syndrome (Inflammation)): A patient has inflammation on a given day if two of the following three criteria are true for that patient on that day:

- the temperature is outside the interval $[36,38]$,

- the patient is mechanically ventilated,

- the white blood count (WBC) $>12.0$ or $<4.0$.

- NEEDS_A_VASOPRESSOR: Vasopressors are medications that are used to increase blood pressure. Severe infection or inflammation are conditions where the peripheral circulation (or bloodvessels) are dilated leading to a decrease in bloodpressure.

- SEVERE-INFlammation: A patient has severe-inflammation on a given day if he has inflammation on that day and at least one of the following three criteria are true for that patient on that day:

- the patient has a kidney-dysfunction,

- the concentration of lactate exceeds $2(\mathrm{mmol} / \mathrm{L})(\mathrm{lac}-$ tate is produced when glucose is burned in the body in the absence of oxygen. An elevated lactate indicates that the body does not receive enough oxygen, either by failure of the cardiovascular system, the respiratory system, or the oxygen carrying capacity of the blood),

- the patient needs a vasopressor.

- inflammation-SHOCK: A patient has inflammationshock on a given day if he has inflammation and needs_a_vasopressor and at least one of the following two criteria is true for that patient on that day:

- the patient has kidney-dysfunction,

- the lactate concentration exceeds $2 \mathrm{mmol} / \mathrm{L}$.

\subsection{Tasks}

Table 1 summarises the learning tasks considered in the preliminary study. Tasks 1 and 2 consider patients that have been in the ICU for one day. Information for this first day as well as admission information are known; the first task is to predict whether the patients will survive or not, and the second task is to predict if their stay in the ICU will be longer than 3 days.

The remaining tasks are to predict whether the patient will be ( $N$ days in the future) in one of the endangering states described in Section 5.2. Admission information as well as information for the current day are known for the patient. Additionally, polynomial curves are fitted to the attribute values of the previous days of the patient's stay and are used to estimate the trend (first derivative evaluated on the current day) and the attribute values $N$ days in the future, these estimates are included as new attributes for the learning algorithms. For these learning tasks exam- 
Table 1

Learning tasks considered in the preliminary study

\begin{tabular}{|c|c|c|}
\hline Task & Examples & Target attribute \\
\hline 1 & Patients (first day) & Survival \\
\hline 2 & Patients (first day) & Length of stay longer than 3 days? \\
\hline 3 & Inflammation-healthy patient-days & Inflammation $N$ days from today? \\
\hline 4 & Severe-inflammation-healthy patient-days & Severe-inflammation $N$ days from today? \\
\hline 5 & Inflammation-shock-healthy patient-days & Inflammation-shock $N$ days from today? \\
\hline 6 & Inflammation-healthy patient-days & Severe-inflammation $N$ days from today? \\
\hline 7 & Inflammation patient-days & Severe-inflammation $N$ days from today? \\
\hline 8 & Inflammation-healthy patient-days & Inflammation-shock $N$ days from today? \\
\hline 9 & Inflammation patient-days & Inflammation-shock $N$ days from today? \\
\hline 10 & Inflammation patient-days & Heal from inflammation $N$ days from today? \\
\hline 11 & Severe-inflammation patient-days & Heal from severe-inflammation $N$ days from today? \\
\hline 12 & Inflammation-shock patient-days & Heal from inflammation-shock $N$ days from today? \\
\hline 13 & Kidney dysfunction-healthy patient-days & Kidney dysfunction $N$ days from today? \\
\hline 14 & Kidney dysfunction patient-days & Heal from kidney dysfunction $N$ days from today? \\
\hline
\end{tabular}

ples are from a particular selection of the patient-day combinations. Inflammation, kidney dysfunction, etc. are conditions which usually last for several days, and therefore a classifier that predicts the same state as today will have a high accuracy for tomorrow without having any useful value. Moreover, doctors may value the correct prediction of a negative and a positive evolution differently. Therefore the learning tasks to predict whether healthy patients will get ill and to predict whether ill patients will heal are considered separately.

\subsection{Methods}

We used four different data mining algorithms on these tasks: Decision trees (DT), First Order Random Forests (FORF), Naive Bayes (NB) and Tree Augmented Naive Bayes (TAN).

The first algorithm is Decision Tree learning (DT) which is of interest because of its symbolic nature. For a general discussion on decision trees, we refer to [28]. For the present study, we used the relational tree learner TILDE (Topdown Induction of Logical Decision Trees) [3] to build regression trees with an $f$-test as stopping criteria. The result is an interpretable tree-shaped model that identifies a small set of attributes that together have high predictive power for the target attribute.

The second algorithm is First Order Random Forests (FORF) [33]. A random forest [4] is a set of decision trees, in our case 33 trees. Each tree is built on a slightly different dataset, obtained by resampling the original dataset. Also, instead of always evaluating all possible tests for a node in the tree (and 'selecting' the best test), some randomness is inserted in the evaluation process: each test has a $20 \%$ probability of being evaluated. When using a random forest for prediction, we average the predictions made by each of the separate trees to get the final prediction. The advantage of using a random forest instead of a single tree is that it removes the influence that small random variations in the data set can have on a learned tree. The random forests in our experiments were built using TILDE according to the algorithm of [33].

The last two algorithms are based on learning Bayesian networks [26]. A Bayesian network is a graphical representation of probabilistic dependencies between attributes. The nodes in the graph represent attributes and edges between attributes indicate direct dependencies (i.e. dependencies not mediated by another attribute). We learned two specific classes of Bayesian networks that are popular for prediction: Naive Bayesian networks (NB) and TreeAugmented Naive Bayesian networks (TAN) [9]. In NB attributes are supposed to be mutually independent given the target attribute (i.e. the only edges are edges from the target attribute to each of the non-target attributes). Because these assumptions are often too strong, TAN allows taking into account certain extra dependencies between non-target attributes by having extra edges (structured as a tree: each non-target attribute has at most one extra parent). It is important to note that in order to obtain good predictions, the presumed dependencies or independencies specified by a Bayesian network do not necessarily have to make sense from a medical point of view.

To automatically learn a NB or TAN, we took a five-step approach. The first two steps were data-preparation steps, while the last three steps effectively constructed a NB or TAN from this data. In the first step, numerical attributes were discretized by splitting the range of each numerical attribute into 5 bins using the 'recursive minimal entropy' algorithm of [7]. In the second step we removed attributes that were not significantly correlated with the target attribute (according to a chi-square test with $p=0.01$ ). In the third step the structure (edges) of the Bayesian network was learned. For NB this step was simple since edges are fixed by definition. For TAN a tree-structured network was learned that captures the most important dependencies between attributes (in addition to the NB edges) using the algorithm of [9]. In the fourth step the probability tables for all attributes were constructed using maximum likelihood estimation with Laplace correction. In a fifth step a transformation of the predicted probabilities was learned 
(by applying isotonic regression [38]) in order to improve calibration since it is known that predictions of NB and TAN are typically poorly calibrated [37] (we define calibration in the next section).

The reason for using the above algorithms is that this way we learn both symbolic, interpretable models (DT,FORF) and more subsymbolic models (NB,TAN). Of these four learners, DT and FORF are relational learners, meaning that they can learn from examples represented in multiple relations (multiple tables in a database). This is important because in this preliminary study already the data is sequential and there is some background knowledge, and more importantly, we expect in the second phase of the project to use a database containing a large number of relations. Since NB and TAN are non-relational learners we used a propositionalisation approach in the preliminary study. For the second phase of our project we plan to use relational Bayesian approaches (see e.g. $[12,11]$ ).

In Appendix A we show some of the learned models to illustrate the typical output of the above algorithms.

\subsection{Evaluation criteria}

To evaluate performance of the different methods described above, we ran each of the methods for each of the tasks using 10-fold cross-validation. We use two evaluation criteria: area under the ROC-curve (AUC) and the Hosmer-Lemeshow statistic.

A ROC-curve is a plot of the false positive rate (FPR) versus the true positive rate (TPR) obtained by thresholding the probabilities predicted by a model, and this for all possible thresholds. The area under the ROC-curve (AUC) is a measure for how well the model can discriminate between positives and negatives. Precisely, the AUC is the probability that. given a randomly selected positive example $p$ and a randomly selected negative example $n, p$ is ranked as being more likely to be positive than $n$. Using AUC as evaluation measure is preferred above accuracy [27], as it allows to trade-off the possibly different costs of incorrectly classifying a positive example as negative and incorrectly classifying a negative example as positive. According to medical standards, an AUC of at least $80 \%$ is required for a model to be valuable [39].

The Hosmer-Lemeshow statistic measures how well calibrated probabilities predicted by a model are. Roughly speaking, probabilities are well calibrated if they correspond to true fractions of positives in the data. In the medical literature the Hosmer-Lemeshow statistic is the standard way of evaluating calibration [18]. The statistic is computed in two steps. In the first step all examples are divided into 10 bins according to their predicted probability of being positive. In the second step it is checked for each bin whether the average predicted probability of being positive for examples in that bin is close to the fraction of positive examples in that bin. This is done by computing a $\chi^{2}$-like measure, which can be translated to a confidence level called the Hosmer-Lemeshow statistic [18]. According to medical standards, this statistic should be at least 0.05 for a model to be acceptable.

AUC and the Hosmer-Lemeshow statistic are complementary evaluation criteria [37]. A model can have perfect discrimination ( $\mathrm{AUC}=100 \%$ ) while being very poorly calibrated, for example when predictions would not be in the range $[0,1]$. Also the opposite holds: a model that is well calibrated can have poor discrimination.

\subsection{Results}

The results are shown in Table 2 (prediction of survival and length of stay), Table 3 (all tasks related to development of endangering states) and Table 4 (all tasks related to recovery from endangering states). For each task we show the total number of examples $N b E x$, the fraction of true positives $p(+)$ and the results for each of the four methods. A result like 79\%/0.69 means that AUC is $79 \%$ and the Hosmer-Lemeshow statistic is 0.69 .

Table 2

Prediction of patient survival and length of stay

\begin{tabular}{lllllll}
\hline Task & NbEx & $p(+)$ & DT & FORF & NB & TAN \\
\hline 1 & 1548 & 0.06 & $79 \% / 0.69$ & $82 \% / 0.69$ & $88 \% / 0.81$ & $86 \% / 0.09$ \\
2 & 1548 & 0.41 & $75 \% / 0.06$ & $79 \% / 0.27$ & $83 \% / 0.10$ & $83 \% / 0.01$ \\
\hline
\end{tabular}

Table 3

Prediction of development of endangering states

\begin{tabular}{clllllll}
\hline Task & $N$ & $N b E x$ & $p(+)$ & DT & FORF & NB & TAN \\
\hline 3 & 1 & 3527 & 0.24 & $66 \% / 0.30$ & $70 \% / 0.59$ & $70 \% / 0.12$ & $73 \% / 0.001$ \\
3 & 2 & 2965 & 0.31 & $63 \% / 0.52$ & $66 \% / 0.70$ & $69 \% / 0.02$ & $70 \% / 0.003$ \\
& & & & & & & \\
4 & 1 & 6871 & 0.08 & $82 \% / 0.55$ & $84 \% / 0.58$ & $82 \% / 0.53$ & $83 \% / 0.35$ \\
4 & 2 & 5772 & 0.10 & $79 \% / 0.36$ & $83 \% / 0.63$ & $81 \% / 0.33$ & $82 \% / 0.09$ \\
4 & 3 & 5131 & 0.12 & $79 \% / 0.32$ & $83 \% / 0.63$ & $81 \% / 0.41$ & $82 \% / 0.11$ \\
& & & & & & & \\
5 & 1 & 9467 & 0.02 & $87 \% / 0.86$ & $91 \% / 0.89$ & $91 \% / 0.59$ & $93 \% / 0.20$ \\
5 & 2 & 8138 & 0.03 & $87 \% / 0.57$ & $91 \% / 0.85$ & $90 \% / 0.58$ & $92 \% / 0.31$ \\
5 & 3 & 7325 & 0.04 & $85 \% / 0.51$ & $90 \% / 0.50$ & $89 \% / 0.33$ & $92 \% / 0.24$ \\
& & & & & & & \\
6 & 1 & 3527 & 0.10 & $86 \% / 0.57$ & $88 \% / 0.84$ & $85 \% / 0.36$ & $87 \% / 0.11$ \\
6 & 2 & 2965 & 0.13 & $81 \% / 0.41$ & $84 \% / 0.61$ & $82 \% / 0.56$ & $84 \% / 0.01$ \\
6 & 3 & 2619 & 0.16 & $79 \% / 0.16$ & $83 \% / 0.48$ & $81 \% / 0.21$ & $84 \% / 0.09$ \\
& & & & & & & \\
7 & 1 & 3344 & 0.05 & $67 \% / 0.95$ & $71 \% / 0.83$ & $80 \% / 0.67$ & $79 \% / 0.23$ \\
7 & 2 & 2807 & 0.07 & $66 \% / 0.67$ & $73 \% / 0.70$ & $79 \% / 0.66$ & $79 \% / 0.40$ \\
7 & 3 & 2512 & 0.08 & $70 \% / 0.32$ & $76 \% / 0.70$ & $78 \% / 0.51$ & $75 \% / 0.26$ \\
& & & & & & & \\
8 & 1 & 3527 & 0.02 & $88 \% / 0.85$ & $95 \% / 0.72$ & $95 \% / 0.64$ & $96 \% / 0.42$ \\
8 & 2 & 2965 & 0.03 & $85 \% / 0.73$ & $91 \% / 0.88$ & $93 \% / 0.35$ & $95 \% / 0.36$ \\
8 & 3 & 2619 & 0.04 & $89 \% / 0.89$ & $92 \% / 0.80$ & $92 \% / 0.77$ & $95 \% / 0.20$ \\
& & & & & & & \\
9 & 1 & 5940 & 0.02 & $81 \% / 0.70$ & $88 \% / 0.91$ & $90 \% / 0.75$ & $92 \% / 0.40$ \\
9 & 2 & 5173 & 0.03 & $87 \% / 0.64$ & $90 \% / 0.60$ & $91 \% / 0.62$ & $90 \% / 0.27$ \\
9 & 3 & 4706 & 0.04 & $82 \% / 0.77$ & $88 \% / 0.82$ & $90 \% / 0.45$ & $91 \% / 0.08$ \\
13 & 2 & 7084 & 0.05 & $82 \% / 0.57$ & $86 \% / 0.57$ & $86 \% / 0.20$ & $88 \% / 0.40$ \\
13 & 3 & 6329 & 0.06 & $84 \% / 0.41$ & $87 \% / 0.20$ & $87 \% / 0.21$ & $88 \% / 0.25$ \\
& 4 & 5790 & 0.07 & $83 \% / 0.25$ & $88 \% / 0.35$ & $87 \% / 0.04$ & $87 \% / 0.05$ \\
\hline
\end{tabular}


Table 4

Prediction of recovery from endangering states

\begin{tabular}{llllllll}
\hline Task & $N$ & NbEx & $p(+)$ & DT & FORF & NB & TAN \\
\hline 10 & 1 & 6756 & 0.25 & $74 \% / 0.10$ & $77 \% / 0.32$ & $75 \% / 0.18$ & $77 \% / 0.21$ \\
10 & 2 & 5937 & 0.29 & $72 \% / 0.14$ & $75 \% / 0.41$ & $72 \% / 0.08$ & $75 \% / 0.02$ \\
11 & 1 & 3412 & 0.25 & $70 \% / 0.26$ & $75 \% / 0.40$ & $72 \% / 0.13$ & $75 \% / 0.02$ \\
11 & 2 & 3130 & 0.33 & $70 \% / 0.12$ & $76 \% / 0.26$ & $72 \% / 0.26$ & $75 \% / 0.07$ \\
11 & 3 & 2913 & 0.37 & $71 \% / 0.05$ & $76 \% / 0.27$ & $71 \% / 0.06$ & $75 \% / 0.03$ \\
12 & 1 & 816 & 0.23 & $70 \% / 0.75$ & $74 \% / 0.39$ & $79 \% / 0.10$ & $80 \% / 0.02$ \\
12 & 2 & 764 & 0.32 & $69 \% / 0.68$ & $73 \% / 0.59$ & $76 \% / 0.09$ & $77 \% / 0.02$ \\
12 & 3 & 719 & 0.38 & $69 \% / 0.78$ & $75 \% / 0.26$ & $80 \% / 0.19$ & $78 \% / 0.12$ \\
14 & 1 & 1942 & 0.08 & $82 \% / 0.72$ & $87 \% / 0.31$ & $80 \% / 0.45$ & $85 \% / 0.43$ \\
14 & 2 & 1818 & 0.11 & $80 \% / 0.13$ & $84 \% / 0.70$ & $78 \% / 0.59$ & $84 \% / 0.30$ \\
14 & 3 & 1715 & 0.13 & $80 \% / 0.46$ & $85 \% / 0.49$ & $80 \% / 0.37$ & $87 \% / 0.20$ \\
14 & 4 & 1624 & 0.15 & $81 \% / 0.39$ & $86 \% / 0.35$ & $81 \% / 0.38$ & $87 \% / 0.12$ \\
\hline
\end{tabular}

\subsection{Discussion}

\subsubsection{Prediction of survival (Task 1)}

Many clinicians use APACHE II scores in order to assess the probability of survival for intensive care patients. This score was developed for survival prediction in critically ill patients, and was empirically derived from large databases of multiple ICUs by applying statistical methods such as logistic regression on admission information and measurements of the first day of patients in the ICU [13]. When using this score to predict survival in our study pop- ulation the obtained AUC was $75 \%$. The models built by our data mining algorithms compare favourably to the APACHE II score, as can be seen in Table 2 for Task 1 .

For some of the patients the database contains predictions made by doctors and nurses about survival. These predictions were made on the basis of the measurements for the first day in ICU of the given patient. Since, for predicting survival, we ran our data mining algorithms also on information for the first day, this gives us the opportunity to compare performance of the models built by data mining with performance of doctors and nurses.

Predictions by doctors and nurses were only available for 908 patients in the database. Since we are not sure whether these patients were selected randomly out of all the 1548 patients, we ran the four data mining algorithms again only on these 908 patients. This allows us to fairly compare performance of the algorithms with that of the doctors and nurses. The resulting ROC-curves (based on 10 -fold cross-validation) for the four algorithms are shown in Fig. 1. The AUC is $65 \%$ for DT, $81 \%$ for FORF, $85 \%$ for NB and $82 \%$ for TAN. Predictions by doctors and nurses are crisp binary predictions (survive or die) and not probabilities of survival or death and hence correspond to single points in ROC-space. These points are also shown in Fig. 1.

We can see that all algorithms except DT perform quite well. For NB and TAN the point for the predictions by
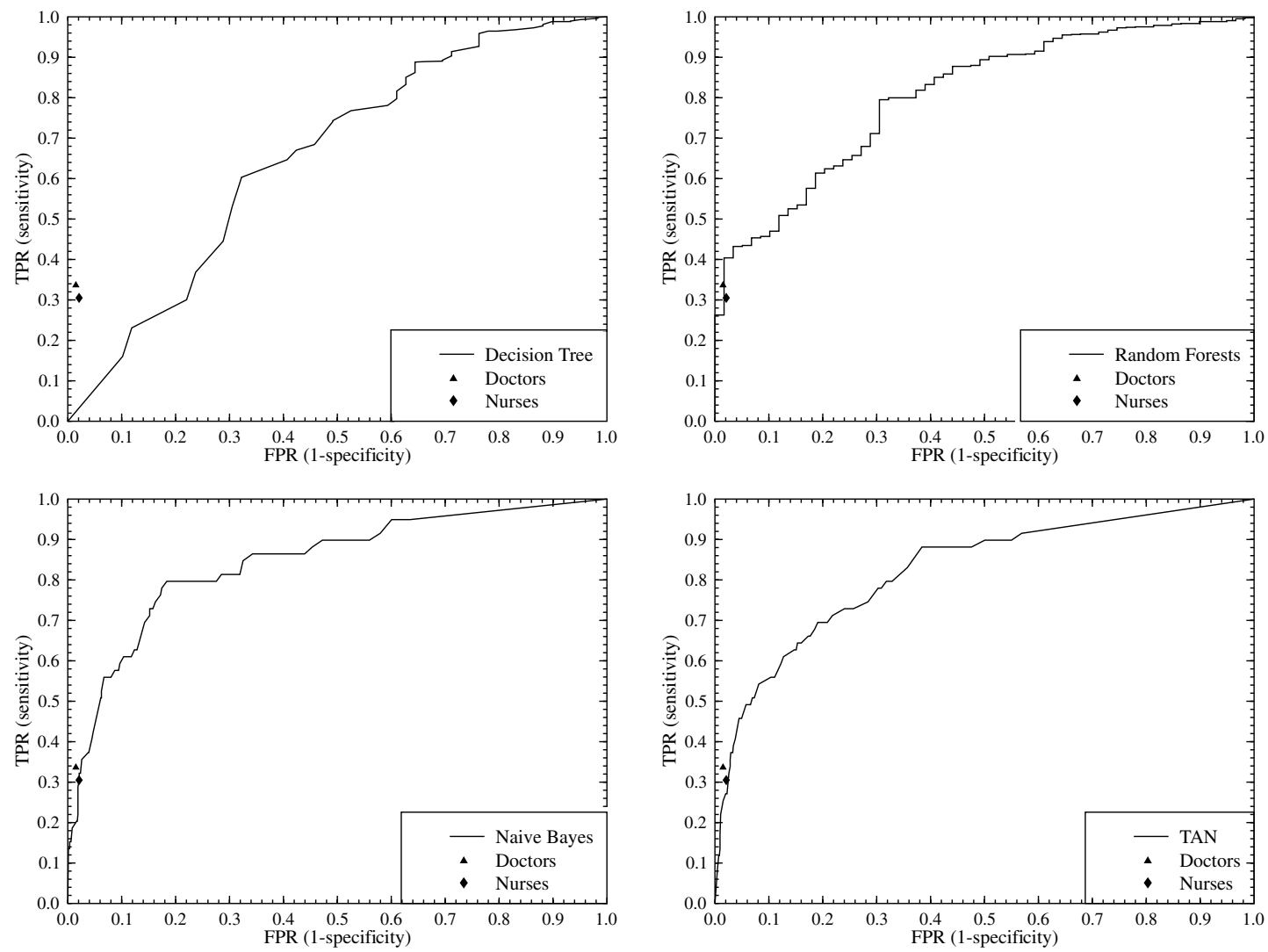

Fig. 1. Predicting survival: comparison of predictions by nurses and doctors and predictions by data mining (for 908 patients). 
nurses is on the ROC-curve while the point for the predictions by doctors is slightly above the ROC-curve (this means NB and TAN perform as good as the nurses and almost as good as the doctors). For FORF both points are on the ROC-curve. These results are very promising since strictly speaking the algorithms had less information available for prediction; in addition to the information registered in the database, the doctors and nurses could also make use of the subjective information of the patient's general appearance.

The top of a number of decision trees were examined by domain experts. They were able to interpret them, and for the tests in most nodes a good 'explanation' could be found in the form of a reasoning that shows how the tested feature indeed influences the expected survival. This provided an additional validation of our learned models. Nevertheless, as was expected the subsymbolic methods outperformed the symbolic method of DT.

\subsubsection{Other tasks}

In predicting the development of endangering states (Table 3), for most of the tasks there was at least one algorithm that performed well according to medical standards. The poor performance for task 3 was expected since prediction of Inflammation is considered a difficult task which doctors cannot perform accurately themselves. Doctors can, on the other hand, accurately predict the development of Kidney Dysfunction one or two days in advance, so it was of interest to see that the algorithms continued to perform well even for predictions 3 or 4 days in advance (Task 13 of Table 3).

For the predictions concerning recovery from endangering states (Table 4), only for Kidney Dysfunction (task 14) were the performances of the models satisfactory within medical standards. This again is in accordance with the medical experts appreciation of there being more difficulty in predicting Inflammation related states. It is also

Table A.1

Attributes recorded upon admission for every patient in the preliminary study

\begin{tabular}{ll}
\hline Age & Admission diagnoses \\
\hline Body length & - Cardiac surgery: \\
Body weight & - Coronary bypass surgery \\
Body mass index & - Heart valve surgery \\
Gender & - Combined surgery \\
Admission postponed & - Intervention for mediastinitis \\
Previously treated in ICU & - Sternum dehiscence \\
Blood glucose level at time of & - Vascular surgery \\
$\quad$ admission & - Thoracic (non-cardiac) surgery \\
Intensive or conventional insulin & - Abdominal surgery \\
$\quad$ therapy & - Neurosurgery or neurological dis- \\
Concurrent disease & ease \\
$\quad$ Immune suppression & - Multiple trauma \\
- Malignancy & - Transplantation \\
$\quad$ - History of diabetes & - Burn injury \\
- History of cardiac disease & - Complications after other surgery \\
\hline
\end{tabular}

noteworthy that the models consistently perform well for task 14 throughout the studied period of 1-4 days in advance.

Note that for the prediction of survival (previous section) we compared performance of the data mining algorithms to performance of the APACHE II score. This was because we tried to predict survival based on the first

Table A.2

Attributes recorded on a daily basis for every patient in the preliminary study

1. ICU pathology
Inflammation
Severe inflammation
Inflammation/shock
Kidney dysfunction
Bacteraemia yes/no
Neuromuscular weakness yes/no:
- Critical illness polyneuropathy (CIPNP) yes/no
- Myopathy yes/no
2. ICU therapy
Vasopressor yes/no
Steroids yes/no
Insulin yes/no
Daily dose of insulin (IU/24 h)*
Daily energy intake (kcal/24 h)
Mechanical ventilation yes/no
Kidney replacement therapy yes/no:
- Continuous veno-venous hemofiltration (CVVH) yes/no
- Intermittent haemodialysis (IHD) yes/no
Plasmapheresis yes/no
Mechanical hemodynamic support yes/no:
- Intra-aortic balloon pump yes/no
- Extra corporeal membrane oxygenation (ECMO) yes/no
- Left ventricular assist device (LVAD) yes/no:
- Medos ${ }^{\text {TM }}$ yes/no
- Novacor ${ }^{\text {TM }}$ yes/no

3. Evaluations

Therapy restriction code Daily APACHE II*

4. Physiological parameters Highest body temperature $/ 24 \mathrm{~h}^{*}$ Lowest body temperature $/ 24 \mathrm{~h}^{*}$ Urine output $/ 24 h^{*}$

5. Laboratory data

Hemoglobin plasma $(\mathrm{g} / \mathrm{dL})$

Hematocrit $(\%)$

Platelet count $\left(10^{9} / \mathrm{L}\right)$

White blood cell count (WBC) $\left(10^{9} / \mathrm{L}\right)$

CRP $(\mathrm{mg} / \mathrm{L})$

Prothrombin Time (PT) $(\%)$

Protein plasma $(\mathrm{g} / \mathrm{L})$

Bilirubin (mg/dL)

Blood glucose level (mg/dL):

- Morning blood glucose level

- Highest blood glucose level/24 h

- Lowest blood glucose level/24 h

Plasma Urea (mg/dL)

Plasma Creatinine $(\mathrm{mg} / \mathrm{dL})$

Urine Creatinine $(\mathrm{mg} / \mathrm{dL})$

Blood Lactate (mmol/L)

Attributes marked with an asterisk $\left(^{*}\right)$ are based on the data of the previous day. 
Table A.3

Decision tree for predicting development severe inflammation 1 day in advance

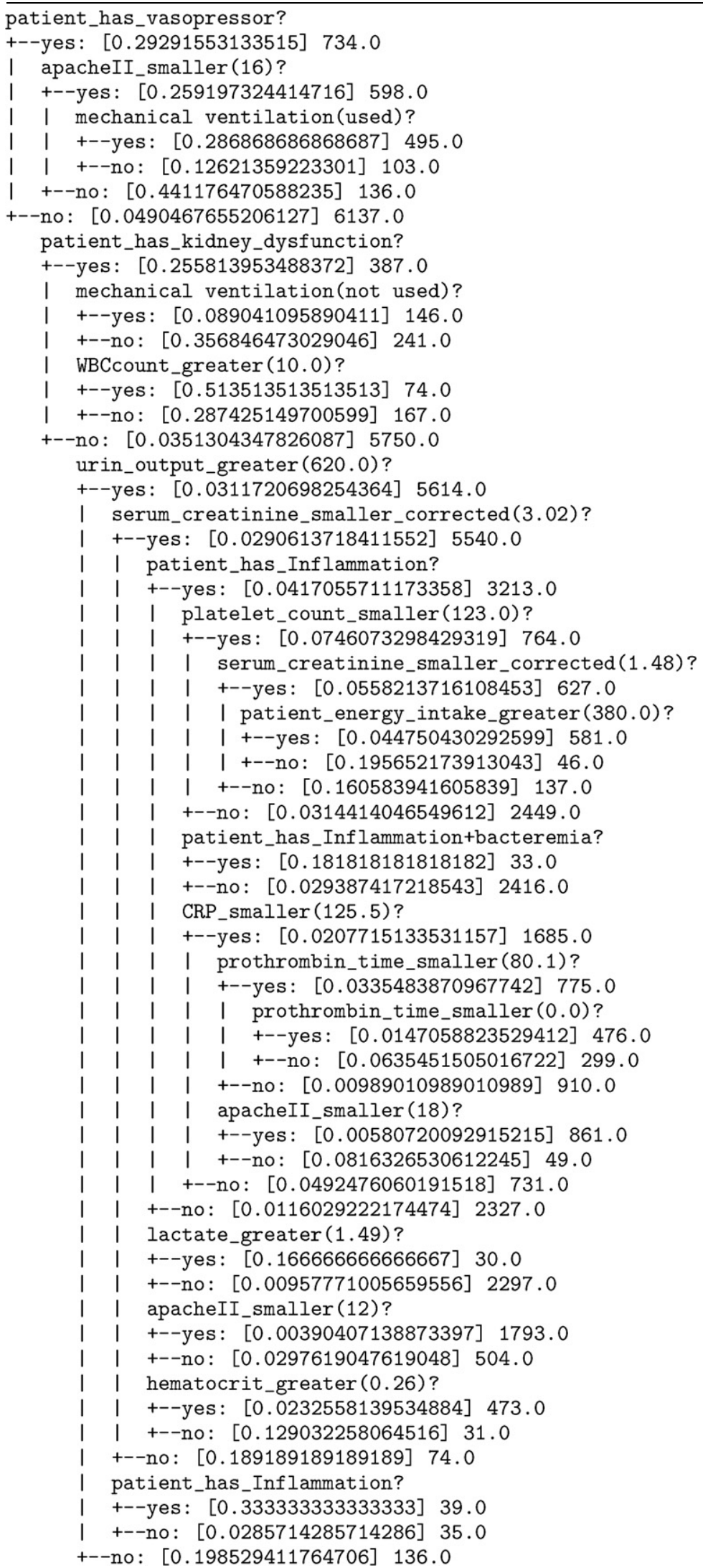


day of the patient in ICU and the APACHE II score was designed exactly for predictions based on the first day. For the endangering states, however, we want predictions on a daily basis (not only after the first day). Hence it was not considered clinically relevant to compare to APACHE II scores here.

The above results show that our methods are promising for the considered predictive tasks within the intensive care context. Therefore, we expect that a more systematic study on a larger amount of data may produce valuable results. Nevertheless, these results also indicate that for certain tasks, such as predicting inflammation, significant improvements are needed further supporting our claim that this is a challenging area for data mining.

\section{Conclusions}

In this paper we described the domain of intensive care. We argued that this application is interesting for data mining and that data mining can significantly progress the state of the art in this and other application domains of engineering. On the one hand it is eligible for data mining since current knowledge is incomplete, there is a strong interest in the field and current data mining technology can produce valuable results. On the other hand it is challenging for data mining researchers since there is need for improvement on current data mining methods in this application domain. In particular, the size of the databases, individual characteristics of every patient, and the presence of different kinds of noise, are aspects that, when combined, make it difficult to extract knowledge from the data.

Consequently, there are two main directions for further work. First, one can use existing data mining techniques to explore more systematically the different prediction tasks one can define in the intensive care area. It is interesting to further extend the list of target variables valuable to clinicians, and to see how difficult they are to tackle with a data mining approach. Second, one can improve the data mining techniques in order to address the problems that currently are still difficult. In particular we believe that relational learning techniques combining expert knowledge and statistical knowledge from data and techniques taking into account the individual characteristics of the patients are important to consider.

Table A.4

TAN for predicting development severe inflammation 1 day in advance

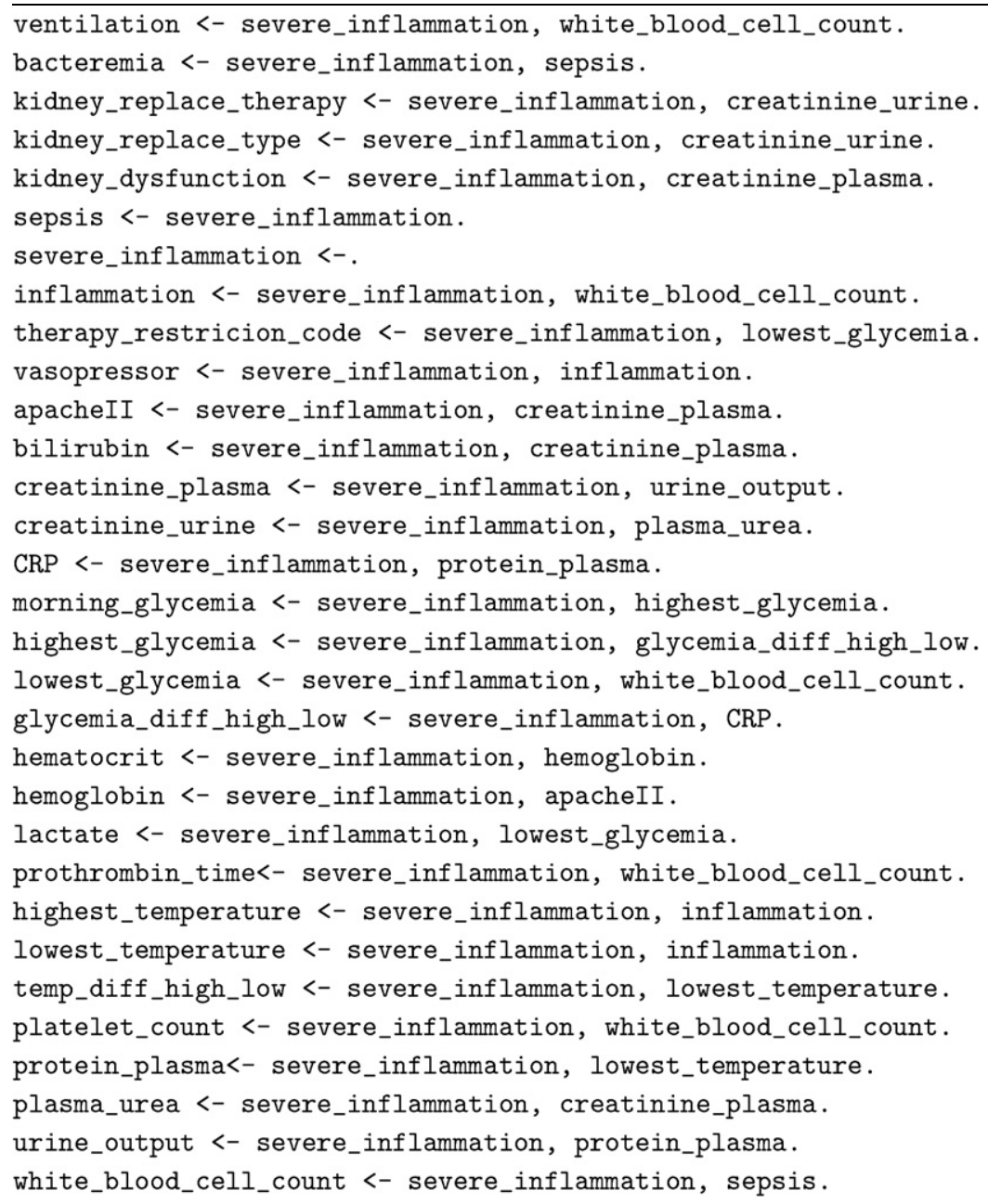


Table A.5

Example of a conditional probability table for TAN network

\begin{tabular}{llll}
\hline Severe_inflammation & White_blood_cell_count & $P$ (mechanical) & $P$ (non-mechanical) \\
\hline No & $\leqslant 12.05$ & 0.817 & 0.183 \\
No & {$[12.05,25.15]$} & 0.691 & 0.309 \\
No & {$[25.15,30.8]$} & 0.762 & 0.238 \\
No & {$[30.8,46.6]$} & 0.850 & 0.150 \\
No & $>46.6$ & 0.250 & 0.750 \\
Yes & $\leqslant 12.05$ & 0.948 & 0.052 \\
Yes & {$[12.05,25.15]$} & 0.701 & 0.299 \\
Yes & {$[25.15,30.8]$} & 0.583 & 0.417 \\
Yes & {$[30.8,46.6]$} & 0.500 & 0.500 \\
Yes & $>46.6$ & 0.667 & 0.333 \\
\hline
\end{tabular}

\section{Appendix A. Illustration of the input data and the learned models for the preliminary study}

In this appendix we illustrate the input data for the preliminary study presented in Section 5 and show some of the learned models.

\section{A.1. The input data}

As explained in Section 5.1, for each patient some attributes were recorded upon admission to the ICU and some attributes were recorded every day during the patient's stay in ICU. We show these attributes in Tables A.1 and A.2 respectively.

\section{A.2. Example of a learned decision tree}

As an example of a typical model learned in the preliminary study of Section 5.1, in Table A.3 we show the decision tree (DT) learned for the prediction of severe inflammation 1 day in advance for patients that are severe inflammation healthy (this corresponds to Task $4, N=1$ in Table 1).

\section{A.3. Example of a learned Bayesian network}

Because the structure of a Naive Bayesian (NB) network is fixed by definition, showing the learned Naive Bayesian networks is not very informative. We only show the structure of a Tree Augmented Naive Bayesian network (TAN) in Table A.4. This network was learnt for the same task as the decision tree of the previous section, i.e. for predicting Severe Inflammation. The notation $X \leftarrow Y, Z$; means that $Y$ and $Z$ are parents of $X$, i.e. there are edges from $Y$ to $X$ and from $Z$ to $X$.

To make the Bayesian network complete we need a conditional probability table for each attribute. As an example we show the table for the attribute 'ventilation' in Table A.5. This attribute has as possible values 'mechanical' and 'non-mechanical' and has as parents the attributes 'severe_inflammation' and 'white_blood_cell_count'.

\section{References}

[1] N. Aoki, M. Wall, J. Demsar, B. Zupan, T. Granchi, M. Schreiber, J. Holcomb, M. Byrne, K. Liscum, G. Goodwin, J. Beck, K. Mattox, Predictive model for survival at the conclusion of a damage control laparotomy, American Journal of Surgery 180 (6) (2000) $540-544$.

[2] R. Bellomo, C. Ronco, J.A. Kellum, R. Mehta, P. Palevsky. The ADQI workgroup, acute renal failure - definition, outcome measures, animal models, fluid therapy and information technology needs, in: the Second International Consensus Conference of the Acute Dialysis Quality Initiative (ADQI) Group, Critical Care, vol. 8(4), August 2004, R204-R212, epub 2004 May 24. <http://ccforum.com/content/ $8 / 4 / \mathrm{R} 204>$.

[3] H. Blockeel, L. De Raedt, Top-down induction of first order logical decision trees, Artificial Intelligence 101 (1-2) (1998) 285-297.

[4] L. Breiman, Random forests, Machine Learning 45 (1) (2001) 5-32.

[5] T.G. Buchman, Nonlinear dynamics, complex systems, and the pathology of critical illness, Current Opinion in Critical Care 10 (2004) 378-382.

[6] L. De Raedt, Attribute-value learning versus inductive logic programming: the missing links (extended abstract), in: D. Page (Ed.), Proceedings of the Eighth International Conference on Inductive Logic Programming, Lecture Notes in Artificial Intelligence, vol. 1446, Springer-Verlag, 1998, pp. 1-8.

[7] J. Dougherty, R. Kohavi, M. Sahami, Supervised and unsupervised discretization of continuous features, in: A. Prieditis, S. Russell (Eds.), Proceedings of the Twelfth International Conference on Machine Learning, Morgan Kaufmann, 1995, pp. 194-202.

[8] S. Džeroski, N. Lavrač (Eds.), Relational Data Mining, SpringerVerlag, 2001

[9] N. Friedman, D. Geiger, M. Goldszmidt, Bayesian network classifiers, Machine Learning 29 (1997) 131-163.

[10] S. Ganzert, J. Guttmann, K. Kersting, R. Kuhlen, C. Putensen, M. Sydow, S. Kramer, Analysis of respiratory pressure-volume curves in intensive care medicine using inductive machine learning, Artificial Intelligence in Medicine 26 (1-2) (2002) 69-86.

[11] L. Getoor, N. Friedman, D. Koller, A. Pfeffer, Learning probabilistic relational models, in: S. Dzeroski, N. Lavrac (Eds.), Relational Data Mining, Springer-Verlag, 2001, pp. 307-334.

[12] K. Kersting, L. De Raedt, Bayesian logic programming: theory and tool, in: An Introduction to Statistical Relational Learning, MIT Press, 2005.

[13] W. Knaus, E. Draper, D. Wagner, J. Zimmerman, APACHE II: a severity of disease classification system, Critical Care Medicine 13 (1985) 818-829.

[14] W. Knaus, D. Wagner, E. Draper, J. Zimmerman, M. Bergner, P. Bastos, C. Sirio, D. Murphy, T. Lotring, A. Damiano, The APACHE III prognostic system. Risk prediction of hospital mortality for critically ill hospitalised adults, Chest 100 (1991) 1619-1636. 
[15] L. Kong, E. Milbrandt, L. Weissfeld, Advances in statistical methodology and their application in critical care, Current Opinion in Critical Care 10 (2004) 391-394.

[16] J. Kreke, A. Schaefer, M. Roberts, Simulation and critical care modeling, Current Opinion in Critical Care 10 (2004) 395-398.

[17] J. Le Gall, P. Loirat, A. Alperovitch, P. Glaser, C. Granthil, D. Mathieu, P. Mercier, R. Thomas, D. Villers, A simplified acute physiology score for ICU patients, Critical Care Medicine 12 (1984) 975-977.

[18] S. Lemeshow, D. Hosmer, A review of goodness of fit statistics for use in the development of logistic regression models, American Journal of Epidemiology 115 (1) (1982) 92-106.

[19] S. Lemeshow, D. Teres, J. Klar, J. Avrunin, S. Gehlbach, J. Rapoport, Mortality probability models (MPM II) based on an international cohort of intensive care unit patients, Journal of the American Medical Association 270 (1993) 2478-2486.

[20] M. Levy, M. Fink, J. Marshall, E. Abraham, D. Angus, D. Cook, J. Cohen, S. Opal, J. Vincent, G. Ramsay, 2001 SCCM/ESICM/ACCP/ ATS/SIS international sepsis definitions conference, Intensive Care Medicine 31 (2003) 1250-1256.

[21] P. Lucas, Bayesian analysis, pattern analysis, and data mining in health care, Current Opinion in Critical Care 10 (2004) 399-403.

[22] G.A. Miller, The magical number seven, plus or minus two: some limits on our capacity for processing information, Psychological Review 63 (1956) 81-97.

[23] K. Morik, P. Brockhausen, T. Joachims, Combining statistical learning with a knowledge-based approach - a case study in intensive care monitoring, in: Proceedings of the 16th International Conference on Machine Learning (ICML-99), Bled, Slovenia, 1999.

[24] K. Morik, M. Imhoff, P. Brockhausen, T. Joachims, U. Gather, Knowledge discovery and knowledge validation in intensive care, Artificial Intelligence in Medicine 19 (3) (2000) 225-249.

[25] S. Moser, W. Jones, S. Brossette, Application of data mining to intensive care unit microbiologic data, Emerging Infectious Diseases 5 (3) (1999) 454-457.

[26] J. Pearl, Probabilistic Reasoning In Intelligent Systems: Networks of Plausible Inference, Morgan Kaufmann Publishers, Inc., San Mateo, California, 1988.

[27] F. Provost, T. Fawcett, R. Kohavi, The case against accuracy estimation for comparing induction algorithms, in: Proceedings of the 15th International Conference on Machine Learning, Morgan Kaufmann, 1998, pp. 445-453.

[28] J.R. Quinlan, C4.5: programs for machine learning Morgan Kaufmann Series in Machine Learning, Morgan Kaufmann, 1993.
[29] L. Rosenberg, Recent innovations in intensive care unit risk-prediction models, Critical Care Medicine 8 (2002) 321-330.

[30] M. Saeed, C. Lieu, G. Raber, R. Mark, Mimic II: a massive temporal icu patient database to support research in intelligent patient monitoring, Computers in Cardiology 29 (2002) 641-644.

[31] B. Sierra, N. Serrano, P. Larranaga, E.J. Plasencia, I. Inza, J.J. Jimenez, P.M.L.M. Revuelta, Using Bayesian networks in the construction of a bi-level multi-classifier. A case study using intensive care unit patients data, Artificial Intelligence in Medicine 22 (3) (2001) 233-248.

[32] G. Van den Berghe, P. Wouters, F. Weekers, C. Verwaest, F. Bruyninckx, M. Schetz, D. Vlasselaers, P. Ferdinande, P. Lauwers, R. Bouillon, Intensive insulin therapy in critically ill patients, New England Journal of Medicine 345 (19) (2001) 1359-1367.

[33] C. Vens, A. Van Assche, H. Blockeel, S. Džeroski, First order random forests with complex aggregates, in: R. Camacho, R. King, A. Srinivasan (Eds.), Proceedings of the 14th International Conference on Inductive Logic Programming, Springer, 2004, pp. 323-340.

[34] M. Verduijn, N. Peek, F. Voorbraak, E. de Jonge, B. de Mol, Dichotomization of ICU length of stay after cardiac surgery based on model precision, in: A. Nowé, T. Lenaerts, K. Steehaut (Eds.), Benelearn 2004: Proceedings of the Annual Machine Learning Conference of Belgium and the Netherlands, 2004, pp. 162-169.

[35] M. Verduijn, N. Peek, F. Voorbraak, E. de Jonge, B. de Mol, Dichotomization of ICU length of stay based on model calibration, in: S. Miksch, J. Hunter, E. Keravnou (Eds.), Proceedings of the 10th Conference on Artificial Intelligence in Medicine (AIME 2005), Springer-Verlag, 2005.

[36] Y. Vodovotz, G. Clermont, C. Chow, G. An, Mathematical models of the acute inflammatory response, Current Opinion in Critical Care 10 (2004) 383-390.

[37] B. Zadrozny, C. Elkan, Obtaining calibrated probability estimates from decision trees and naive bayesian classifiers, in: Proceedings of ICML-2001 - Eighteenth International Conference on Machine Learning, Morgan Kaufmann, 2001, pp. 609-616.

[38] B. Zadrozny, C. Elkan, Transforming classifier scores into accurate multiclass probability estimates, in: Proceedings of the Eighth ACM SIGKDD International Conference on Knowledge Discovery and Data Mining (KDD’02), ACM, 2002, pp. 694-699.

[39] J. Zimmerman, A. Kramer, D. McNair, F. Malila, Acute physiology and chronic health evaluation (APACHE) IV: hospital mortality assessment for today's critically ill patients, Critical Care Medicine 34 (5) (2006) 1297-1310. 